\title{
Operator behavior in a dynamic visual signal detection task'
}

\author{
ROBERT M. GLORIOSO, ${ }^{2}$ U. S. ARMY ELECTRONICS COMMAND, FT. MONMOUTH, N.J. \\ ROBERT M. LEVY, ${ }^{3}$ GENERAL DYNAMICS, ELECTRIC BOAT DIVISION
}

The effects of changes in signal-to-noise ratio $(S / N)$ and feedback state on the operator's detection behavior, particularly derived operator thresholds, were investigated using a discrete visual signal. It was found that: (1) the operator behaves as if he has established different thresholds as a function of $S / N ;(2)$ his decision thresholds are relatively independent of the presence or absence of feedback; and (3) the operator will change his threshold accordingly for a change from one $S / N$ to another. $A$ model of the operator's behavior, which introduced the concept of "operator" noise, yielded predictions of detection and false alarm probabilities which were extremely close to the observed values.

Signal detection theory has provided a strong foundation for analyzing a wide range of psychological problems (Green \& Swets, 1966). An important finding is the recognition of the variable nature of the human decision criterion or threshold. Some parameters that have been found to affect the operator's decision criteria are a priori event probability and cost functions (Green \& Swets, 1966), and signal-to-noise ratio (Brazeal \& Booth, 1966). Most studies, however, have been concerned either with deriving Receiver Operating Characteristics (ROC) curves or with measuring the threshold as a function of signal-to-noise ratio, $\mathrm{S} / \mathrm{N}$, under static conditions. That is, the stimulus conditions are constant throughout each experimental run and the data are analyzed with respect to these fixed stimulus parameters. The present research asks questions about the nature of the decision criterion under dynamic conditions; i.e., the stimulus conditions change within each experimental run and the data are analyzed with respect to the change in stimulus conditions. Static conditions are, in fact, special cases of the dynamic condition; therefore, the extension of research to dynamic conditions is an important generalization for the development of a model to describe the behavior of the operator in the signal detection task.

Green and Swets $(1966$, p. 180) state, ". . the comparison of variation in human sensory performance with explicit, quantitative models of optimal detection systems is an important and useful tool of analysis." Classical signal detection tasks are not usually analyzed with respect to an optimum detector since the experimental data are not easily interpreted in these terms. A basic difficulty is to select a task in which the operator's behavior can be compared to optimum behavior. The task used in the present research is one in which the experimental data are directly interpretable in terms of the optimum detector.

The task is the detection of targets in a discrete visual noise field such as the simulated sonar Bearing-Time display shown in

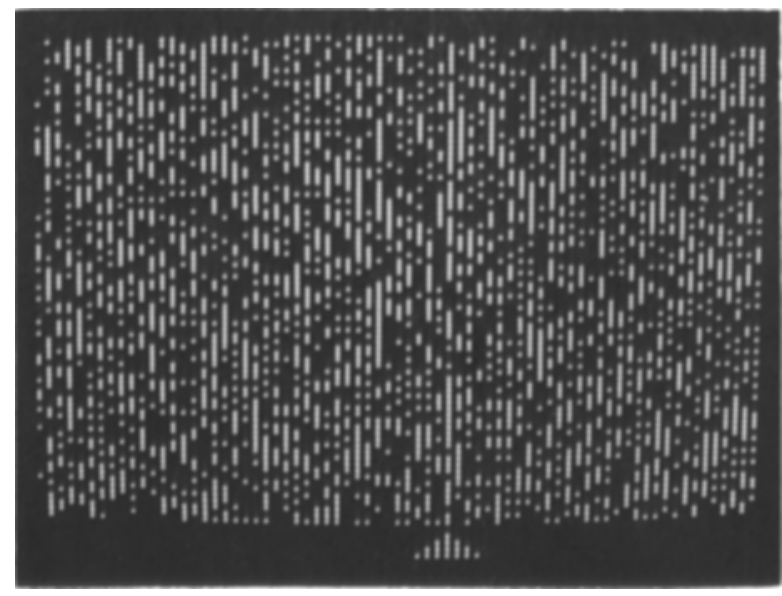

Fig. 1. Simulated sonar Time-Bearing display.

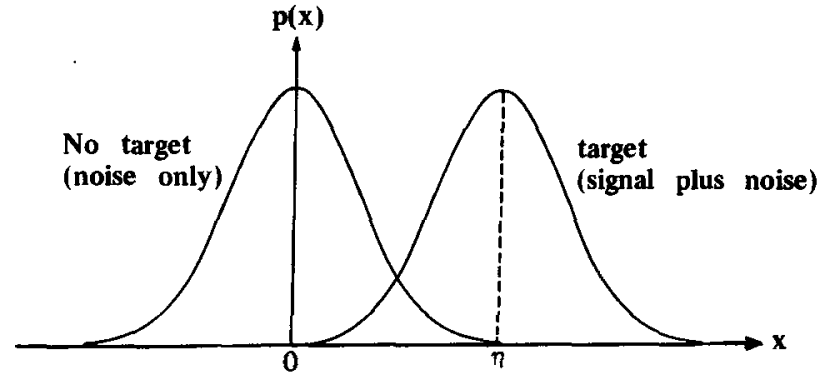

Fig. 2. Distributions at input to display generating apparatus.

Fig. 1. If a target is present, it always appears in the column indicated by the arrow. The display has 80 rows and 72 columns and is $10 \mathrm{in}$. wide $\times 7 \mathrm{in}$. high.

When the total possible number of intensified points in a column is 80 , the expected number of intensified points in the column is 40 if no target is present and the expected number of intensified points is greater than 40 if a target is present. The amount that the expected number is greater than 40 is a function of the relative strength of the target as given by the $\mathrm{S} / \mathrm{N}$. The oeprator indicates his decision as to the presence or absence of a target in the indicated column based on the number, or some function of the number, of intensified points in the column. Feedback (knowledge of results) can be supplied by changing the arrow at the bottom of the display to an $\mathrm{H}$ (for a hit) if the operator's decision is correct and to an $M$ (for a miss) if the decision is incorrect. In order to understand the task more clearly it is helpful to discuss the generation of these displays.

The display apparatus samples either the noise or signal-plusnoise distribution in increments of time (Brazeal \& Booth, 1965, 1966). The samples are independent. When no target is present, the samples, $\mathrm{p}_{\mathrm{nt}}(\mathrm{x})$, are Gaussian (normally) distributed with zero mean and unit standard deviation

$$
p_{n t}(x)=n(0,1)=\frac{1}{\sqrt{2 \pi}} \exp \frac{-x^{2}}{2}
$$

When a target is present, the samples, $p_{t}(x)$ are Gaussian distributed with positive mean $\eta$ and unit standard deviation

$$
p_{t}(x)=n(\eta, 1)=\frac{1}{\sqrt{2 \pi}} \exp \frac{-(x-\eta)^{2}}{2}
$$

Figure 2 illustrates the relationship between these two distributions. The sample amplitudes are then clipped about zero by an infinite clipper and the resulting binary sequency $y_{i}, i=1,2 \ldots$ constitutes the information contained in the display. The output of the clipper is 1 if the sample amplitude is greater than zero and is 0 if the sample amplitude is less than zero. A one is indicated by an intensified point on the display. Thus, when the noise alone or no-target distribution, NT, is sampled, the probabilities that a point will be intensified, $P_{\mathbf{0}}$, or will not be intensified, $Q_{0}$ are:

$$
\mathrm{P}\left(\mathrm{y}_{\mathrm{i}}=1 \text { i NT }\right)=\int_{0}^{\dot{\infty}} \mathrm{n}(0,1) \mathrm{dx}=0.5=\mathrm{P}_{0}=\mathrm{Q}_{0}
$$

When the signal-plus-noise distribution, $S+N T$, is sampled, the probability that a point will be intensified, $P_{1}$ is greater than the probability of not being intensified, $Q_{1}$. The probabilities $P_{1}$ and $\mathrm{Q}_{1}$ are given by

$$
\mathrm{P}\left(\mathrm{y}_{\mathrm{i}}=1 \mathrm{IS}+\mathrm{NT}\right)=1-\mathrm{P}\left(\mathrm{y}_{\mathrm{i}}=0 \mathrm{IS}+\mathrm{NT}\right)=\int_{0}^{\infty} \mathrm{n}(\eta, 1) \mathrm{dx}
$$

where $\eta$ is the signal-to-noise ratio and $P\left(y_{i}=1 \mid S+N T\right)=P_{1}$ and 
Table 1

Dipplay Parameters as a Function of S/N

\begin{tabular}{rcccc} 
S/N & $P_{1}$ & $\begin{array}{c}\text { Expected } \\
\text { Value }\left(80 P_{1}\right)\end{array}$ & $\begin{array}{c}\text { Standard } \\
\text { Deviation }\end{array}$ & $\begin{array}{c}\text { Optimum } \\
\text { Decision } \\
\text { Threshold }\end{array}$ \\
\hline$-14.5 \mathrm{~dB}$ & .57 & 46 & 4.4 & 43 \\
$-8.5 \mathrm{~dB}$ & .65 & 52 & 4.3 & 46 \\
$-3.5 \mathrm{~dB}$ & .75 & 60 & 3.9 & 50 \\
\hline
\end{tabular}

$P\left(y_{i}=0, S+N T\right)=Q_{1}$. The number of intensified points in a column of the display is binomially distributed. If no target is present, the expected number of intensified points in the column is

$$
\bar{N}_{1}=N_{0}=80 \times .5=40
$$

and the standard deviation of the distribution is

$$
\sigma_{0}=\sqrt{\mathrm{NP}_{0} \mathrm{Q}_{0}}=\sqrt{20}
$$

If a target is present (samples taken from the signal-plus-noise distribution), the expected number of intensified points in a column is greater than 40 , and the standard deviation is less than $\sqrt{ } 20$. The amount that the expected number of intensified points exceeds 40 if a target is present is a function of $P_{1}$ which, in turn, is a function of the $S / \dot{N}$. Thus, the number of intensified points in a column is binomially distributed and the expected number of intensified points is a direct function of the $S / N$.

The operator's task is to indicate whether or not he thinks a target is present in the indicated column This task can be accomplished by using an optimum decision strategy as defined by the likelihood ratio, L(x) (Brazeal \& Booth, 1966), where

$$
L(x)=\frac{P_{1} N_{1} Q_{1}^{N}-N_{1}}{P_{0} N_{1} Q_{0} N-N_{1}}
$$

$N_{1}$ is the number of intensified points in the target column. But, for this task, $P_{0}=Q_{0}=1 / 2$, therefore

$$
L(x)={ }_{2} N_{P_{1}} N_{1}\left(1-P_{1}\right)^{N-N_{1}}
$$

Thus, given the $S / N$, which specifies $P_{1}$, and $N_{1}$, the likelihood ratio can be computed. If the computed value of $L(x)$ is compared to a threshold, the decision is made as follows:

if $L(x) \geqslant L_{T}$, decide target

if $L(x)<L_{T}$, decide no target

The threshold value, $L_{T}$, for minimum expected risk is given by (Selin, 1965)

$$
\mathrm{L}_{\mathrm{T}}=\frac{\pi_{0} \mathrm{C}_{0}}{\pi_{1} \mathrm{C}_{1}}
$$

where $\pi_{0}$ and $\pi_{1}$ are the a priori occurrence probabilities of a target and no target, respectively, and $C_{0}$ and $C_{1}$ are the total costs associated with a target and a no target decision, respectively. If, as in the present study, the a priori target occurrence probability is fixed at 0.5 and the decision costs are assigned equal weights, $L_{T}$ equals one. When $L(x)$ is set equal to one, the optimum threshold can be found in ternis of the number of intensified points in the target column, $N_{1}$, by taking logs of Equation 8. For this condition $N_{1}-T$, the optimum threshold, and is given by

$$
T=-N \frac{\log 2 Q_{1}}{\log \left(P_{1} / Q_{1}\right)}
$$

The decision rule now is:

if $\mathrm{N}_{1} \geqslant T$, decide target

if $N_{1}<T$, decide no target
Note that the optimum threshold, T, for this task can be defined as a function of the signalto-noise ratio only.

Although there are two aspects to dynamic detection behavior, the transient and steady state responses, the present research is concerned with the steady state response following a transition in the value of the stimulus parameters. The parameters which were varied were $S / N$ and feedback. The $S / N$ parameter was chosen since it has the largest effect, both empirically and theoretically, on detection. Also, when all other input parameters are fixed, the optimum detector can be specified as a function of the $S / N$ alone. Feedback is not one of the input parameters affecting the optimum detector; however, its effects on the human's detection processes are of fundamental importance. Since the optimum detector is specified in terms of thresholds as a function of $S / N$, the interpretation of the operator's behavior in terms of decision thresholds derived from the operator's performance is of interest.

The goals of the present research are to answer three basic questions about the operator's behavior in a dynamic detection task and to develop a model which describes this behavior. The three questions are: (1) Can stable operator decision thresholds be trained for different S/Ns? (2) Are operator thresholds stable regardless of the presence or absence of feedback? (3) Given a set of $S / N s$ to which the operator has been trained, will a change from $(\mathrm{S} / \mathrm{N}) \times$ to $(\mathrm{S} / \mathrm{N}) \mathrm{y}$ cause the operator to change his threshold from that trained to $(\mathrm{S} / \mathrm{N}) \mathrm{x}$ to that trained to $(\mathrm{S} / \mathrm{N}) \mathrm{y}$ ?

\section{Experimental Conditions}

\section{METHOD}

The three questions above were investigated in a single integrated experiment. The first question was investigated by comparing thresholds derived from the data of trained Ss at different $\mathrm{S} / \mathrm{Ns}$ with and without feedback. The second question was investigated under constant intrasession $S / N$ where, however, the feedback state was changed either from feedback to no feedback or vice versa. The operator's thresholds under the two feedback states were then compared. The third question was studied under conditions of constant intrasession feedback but where the $\mathrm{S} / \mathrm{N}$ was changed. The derived thresholds were again compared.

Three $\mathrm{S} / \mathrm{Ns}$ were used in order to guarantee that the operator be uncertain about the new $S / N$ following a change in $S / N$. The three values of $\mathrm{S} / \mathrm{N},-3.5 \mathrm{~dB},-8.5 \mathrm{~dB}$, and $-14.5 \mathrm{~dB}$, were chosen since they provide a good spacing among the optimum thresholds, and operator detection and false alarm probabilities are well separated. The point intensification probability, $P_{1}$, the expected number of intensified points in a column, the standard deviation of the column count, and the optimum decision thresholds associated with the three S/Ns are given in Table 1 . The 24 experimental conditions used to answer the three questions are summarized in Table 2. As indicated in Table 2, an experimental session consisted of 144 trials where displays were generated independently for each trial. Except for Conditions $1-6$ in which the $S / N$ and feedback state were constant throughout, Trials 1-72 were at some Condition A and Trials 73-144 at some Condition B. Trials 1-12 and 73-84 were treated as practice.

\section{Apparatus}

The experimental apparatus consisted of a PDP-S digital processor with external logic, noise generator, pushbutton matrix, and Fairchild 737A, 17-in. cathode ray display with normal persistence phosphor (P-31). The display was enclosed in a relatively light-proof, sound-proof cubicle, $7 \mathrm{ft}$ high $x 4 \mathrm{ft}$ wide $x$ $6 \mathrm{ft}$ long. The overall system is described in detail elsewhere (Booth, Glorioso, Levy, Walter, \& Kaufman, 1967; Brazeal \& Booth, 1965).

\section{Procedure}

A trial began when the operator pushed a button which turned on the display. He then indicated his decision as to the presence or absence of a signal in the column indicated by the arrow by pushing the appropriate button. Under feedback conditions, the arrow was replaced by $\mathrm{H}$ or $\mathrm{M}$, indicating correct or incorrect 
Table 2

Experimental Conditions

\begin{tabular}{|c|c|c|c|c|}
\hline \multirow{3}{*}{$\begin{array}{l}\mathrm{S} / \mathrm{N} \text { and feedback } \\
\text { dynamics }\end{array}$} & \multicolumn{4}{|c|}{ Trials } \\
\hline & \multicolumn{2}{|c|}{$1-72$} & \multicolumn{2}{|c|}{$73-144$} \\
\hline & $\mathrm{S} / \mathrm{N}^{\mathrm{a}}$ & $\mathrm{FB}^{\mathrm{b}}$ & $\mathbf{S} / \mathbf{N}$ & FB \\
\hline \multirow{6}{*}{$\begin{array}{l}\text { S/N constant, } \\
\text { feedback state } \\
\text { constant }\end{array}$} & $\mathbf{H}$ & 1 & $\mathbf{H}$ & 1 \\
\hline & $\mathbf{M}$ & 1 & $\mathbf{M}$ & 1 \\
\hline & $\mathbf{L}$ & 1 & $\mathbf{L}$ & 1 \\
\hline & $\mathbf{H}$ & 0 & $\mathrm{H}$ & 0 \\
\hline & $\mathbf{M}$ & 0 & $\mathbf{M}$ & 0 \\
\hline & $\mathbf{L}$ & 0 & $\mathbf{L}$ & 0 \\
\hline \multirow{6}{*}{$\begin{array}{l}\text { Constant feedback } \\
\text { S/N change }\end{array}$} & $\mathbf{H}$ & 1 & $\mathbf{M}$ & 1 \\
\hline & $\mathbf{H}$ & 1 & $\mathbf{L}$ & 1 \\
\hline & $\mathbf{M}$ & 1 & $\mathbf{L}$ & 1 \\
\hline & $\mathbf{M}$ & 1 & $\mathbf{H}$ & 1 \\
\hline & L & 1 & $\mathbf{H}$ & 1 \\
\hline & $\mathrm{L}$ & 1 & $\mathbf{M}$ & 1 \\
\hline \multirow{6}{*}{$\begin{array}{l}\text { Constant no-feedback } \\
\text { S/N change }\end{array}$} & $\mathrm{H}$ & 0 & $\mathbf{M}$ & 0 \\
\hline & $\mathrm{H}$ & 0 & $\mathbf{L}$ & 0 \\
\hline & $\mathbf{M}$ & 0 & $\mathbf{L}$ & 0 \\
\hline & $\mathbf{M}$ & 0 & $\mathbf{H}$ & 0 \\
\hline & $\mathbf{L}$ & 0 & $H$ & 0 \\
\hline & L & 0 & $\mathbf{M}$ & 0 \\
\hline \multirow{6}{*}{$\begin{array}{l}\text { S/N constant, } \\
\text { feedback state } \\
\text { change }\end{array}$} & $\mathbf{H}$ & 1 & $\mathbf{H}$ & 0 \\
\hline & $\mathbf{M}$ & 1 & $\mathbf{M}$ & 0 \\
\hline & L & 1 & $\mathbf{L}$ & 0 \\
\hline & $\mathbf{H}$ & 0 & $\mathbf{H}$ & 1 \\
\hline & $\mathbf{M}$ & 0 & $\mathbf{M}$ & 1 \\
\hline & $\mathrm{L}$ & 0 & L & 1 \\
\hline
\end{tabular}

$a_{S / N}: H=-3.5 \mathrm{~dB}, M=-8.5 \mathrm{~dB}, L=-14.5 \mathrm{~dB}$

$b_{\text {FB: } 0}=$ no-feedback, $1=$ feedback

deicision, respectively; under no-feedback conditions, the arrow remained after the decision was made. After the decision was made, the complete display remained on for several seconds. The feedback information was based on the distribution from which the points in the indicated column were sampled and not the number of points in it. Thus, with 39 points in the column, it was possible for a response of "no target" to be incorrect. Although Ss knew the overall set of S/Ns, they did not know the specific S/Ns or feedback states to be viewed within any given experimental session. Trial-by-trial data were printed and punched by the computer.

Four well-trained male Ss participated in the experiment. Three Ss viewed the set of 24 conditions once and the fourth $S$ viewed the set of conditions twice. The conditions were presented in a different random order for each $S$. Each experimental session lasted about $20 \mathrm{~min}$. No $\mathrm{S}$ participated in more than two sessions per day.

The Ss were instructed to weigh false alarms and false dismissals equally and that the a priori target occurrence probability was 0.5 . In order to insure that Ss were not biased in their establishment of response thresholds, they were not told the values of the optimum decision thresholds for the different $\mathrm{S} / \mathrm{Ns}$. The Ss were told, when making a decision, to take only as much time as needed to make an unhurried and not careless decision.

\section{RESULTS AND DISCUSSION}

The first data analysis is concerned with derived decision thresholds. In order to derive these thresholds, a set of tables was generated using the discrete ROC concept (Brazeal \& Booth, 1966) which is based on the cumulative probabilities of two binomial distributions for fixed $\mathrm{S} / \mathrm{Ns}$. These distributions are the same ones used in the calculation of the optimum detector. The use of these distributions to derive equivalent operator thresholds assumes that the operator's decision process is noiseless, i.e., that
Table 3

Derived Operator Thresholds as Functions of $\mathrm{S} / \mathrm{N}$ and Feedback

\begin{tabular}{|c|c|c|c|c|c|c|c|c|c|}
\hline \multirow[b]{2}{*}{ Question } & \multicolumn{3}{|c|}{$-3.5 \mathrm{~dB}$} & \multicolumn{3}{|c|}{$-8.5 \mathrm{~dB}$} & \multicolumn{3}{|c|}{$-14.5 \mathrm{~dB}$} \\
\hline & FB & No $F B$ & Pooled & FB & No FB & Pooled & FB & No FB & Pooled \\
\hline 1 & 47.7 & 48.8 & 48.2 & 45.5 & 44.9 & 45.2 & 42.5 & 42.8 & 42.7 \\
\hline 2 & 47.0 & 48.6 & 47.7 & 45.0 & 45.0 & 45.0 & 42.9 & 42.8 & 42.9 \\
\hline 3 & 47.8 & 47.7 & 47.7 & 44.9 & 45.9 & 45.4 & 42.4 & 43.8 & 43.1 \\
\hline Optimum & & & 50 & & & 46 & & & 43 \\
\hline
\end{tabular}

the subjective and input distributions are the same. These tables give detection and false alarm probabilities as a function of threshold for the input S/Ns. The operator decision threshold can be derived from the false alarm probability which is a function of the noise distribution alone and not of the signal-plus-noise distribution.

The derived thresholds used to answer the three questions raised above are summarized in Table 3. These data were obtained by pooling false alarms over Ss and over the appropriate conditions since inter-subject and inter-condition variability was small and not systematic. The number of samples used to estimate false alarm probabilities for either feedback or no-feedback conditions was 300 for Question 1, 225 for Question 2, and 500 for Question 3. It is readily apparent that the operator behaves as if he has established different thresholds for each $\mathrm{S} / \mathrm{N}$. These thresholds are relatively unaffected by the presence or absence of feedback or by an intrasession change in feedback state. However, following changes in $\mathrm{S} / \mathrm{N}$, the operator changes his decision threshold accordingly. This is shown more clearly in Table 4 in which the derived decision thresholds are given as a function of the change in $\mathrm{S} / \mathrm{N}$. Thus, it appears that in this task, $\mathrm{S} / \mathrm{N}$ is the prime determinant of the operator's decision threshold. It is noteworthy that the distributions of thresholds are nonoverlapping as a function of $\mathrm{S} / \mathrm{N}$ and, moreover, they are very close to the analytically determined optimum thresholds.

The results presented thus far provide, to some degree, satisfactory answers to the questions posed earlier and the basis from which a threshold model can be developed. However, the assumption of a "noiseless" operator is certainly restrictive and ignores basic characteristics of the operator's decision processes. For example, if the discrete ROC curves are entered for the input $\mathrm{S} / \mathrm{Ns}$ with the thresholds derived above, the corresponding detection probabilities tend to overestimate the observed operator detection probabilities. Thus, a threshold model which assumes that the operator's decision process is noiseless is inadequate.

Input-output relationships for noiseless and noisy decision processes are illustrated in Fig. 3. A noiseless decision is represented by a threshold where the input is a sample from either the noise or signal-plus-noise distribution and the output is a "target" decision if, and only if, the sample value equals or exceeds the threshold, $T$. The decision process model can be made noisy by adding noise to the input sample or by moving the threshold randomly about the mean value, $T$, from trial to trial. In the noisy input-output relationship illustrated in Fig. 3 the perturbations were assumed to be normally distributed. For the present discussion the model in which noise is added to the input sample as shown in Fig. 4 will be used. The added noise is called "operator" noise. This noise model is essentially the same one

Table 4

Thresholds after S/N Change-pooled Feedback and No Feedback

\begin{tabular}{cccc} 
S/N-first half & \multicolumn{3}{c}{ S/N-second half } \\
\cline { 2 - 4 } & $-3.5 \mathrm{~dB}$ & $-8.5 \mathrm{~dB}$ & $-14.5 \mathrm{~dB}$ \\
\hline$-3.5 \mathrm{~dB}$ & - & 45.3 & 43.6 \\
$-8.5 \mathrm{~dB}$ & 47.3 & - & 42.6 \\
$-14.5 \mathrm{~dB}$ & 48.0 & 45.0 & - \\
\hline
\end{tabular}




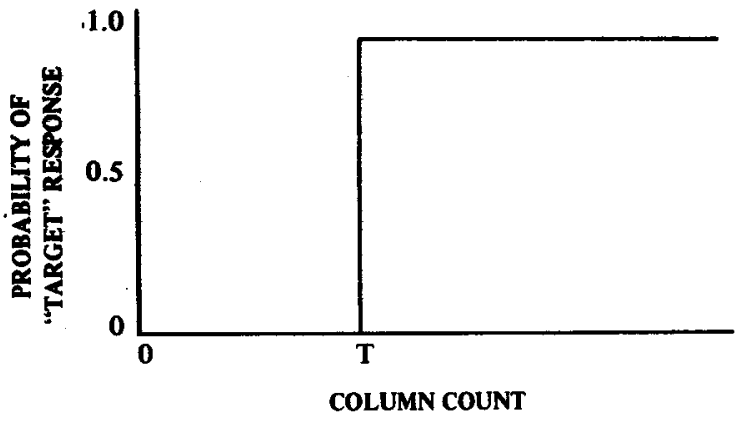

Input-Output Relationship-Noiseless Decision Process

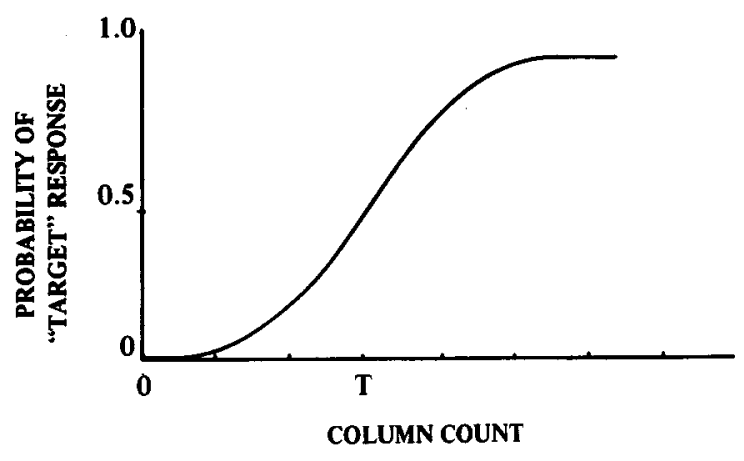

Input-Output Relationship-Noisy Decision Process

Fig. 3. Input-output relationships, noiseless and noisy decision processes.

proposed by Brazeal and Booth (1966). A measure of the operator noise is the standard deviation of the input-output relationship, $\sigma$ op. If the cumulative distribution of the input-output relationship is normal, $\sigma$ op is equal to one-half the interval between .16 and .84 "target" response proabbilities and the threshold $\mathrm{T}$ is the column count at the .50 target response probability.

For each $S / N$ and feedback condition, pooled over all $S s$, the probability of responding "target" was plotted as a function of column count. The number of samples at each count were approximately 110 for $-8.5 \mathrm{~dB}$ and $-14.5 \mathrm{~dB}$ but only 25 samples per point in the region of 47 to 54 column counts and 50 samples or more elsewhere for $-3.5 \mathrm{~dB}$. The paucity of samples in the middle range for $-3.5 \mathrm{~dB}$ was due to the larger separation between the noise and signal-plus-noise distributions at this $S / N$. The psychometric functions (probability of "target" response vs column count) as a function of feedback state are shown in Figs. 5,6 , and 7 for $-14.5 \mathrm{~dB},-8.5 \mathrm{~dB}$, and $-3.5 \mathrm{~dB}$, respectively. The psychometric functions derived by pooling both feedback states as a function of $\mathrm{S} / \mathrm{N}$ are given in Fig. 8. The data given in Figs. 5-8 are fit by a normal distribution.

The measures of $\sigma$ op and $\mathrm{T}$ derived from the functions shown in Figs. 5-7 are shown in Table 5. The values of operator noise appear to be relatively constant as a function of $\mathrm{S} / \mathrm{N}$ and relatively independent of the feedback state. The average value of $\sigma$ op is 3.15 which is equivalent to a loss in $\mathrm{S} / \mathrm{N}$ of $1.6 \mathrm{~dB}$ with respect to a noiseless decision process. The new derived thresholds are very close to the analytically determined optimum thresholds. These

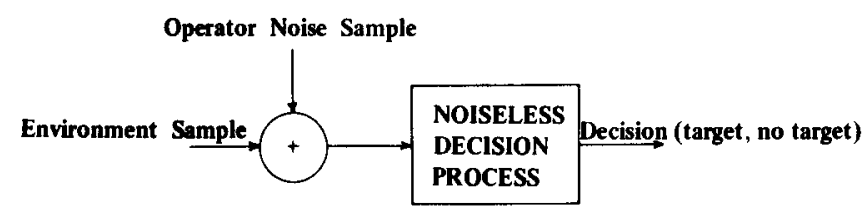

Fig. 4. Additive operator noise representation.

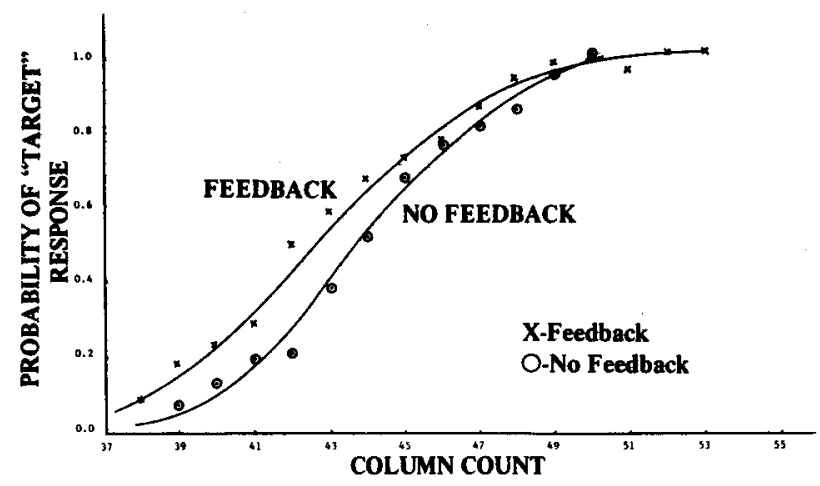

Fig. 5. Psychometric functions: $-14.5 \mathrm{~dB}$.

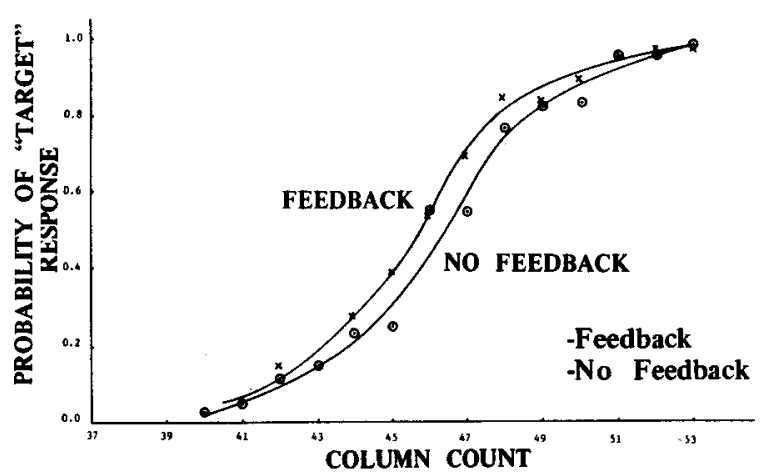

Fig. 6. Psychometric functions: $-8.5 \mathrm{~dB}$.

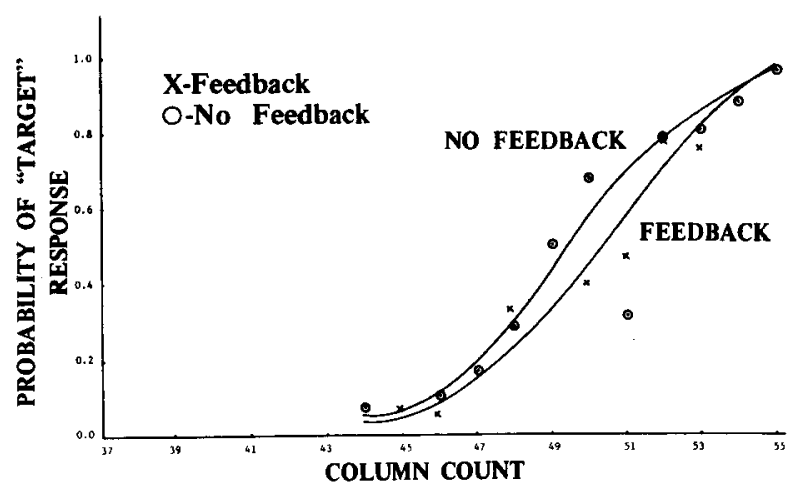

Fig. 7. Psychometric functions: $-\mathbf{3 . 5} \mathbf{d B}$.

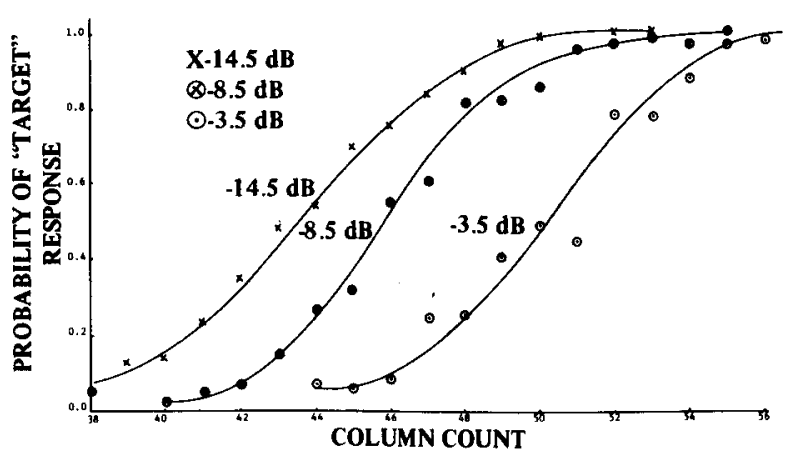

Fig. 8. Psychometric functions: pooled feedback and no feedback. 
Table 5

Derived Operator Characteristics as Functions of $\mathbf{S} / \mathrm{N}$ and Feedback (from Psychometric Functions)

\begin{tabular}{|c|c|c|c|c|c|c|}
\hline \multirow{2}{*}{$\begin{array}{c}\text { Operator } \\
\text { Characteristics }\end{array}$} & \multicolumn{2}{|c|}{$-3.5 \mathrm{~dB}$} & \multicolumn{2}{|c|}{$-8.5 \mathrm{~dB}$} & \multicolumn{2}{|c|}{$-14.5 \mathrm{~dB}$} \\
\hline & FB & No FB & FB & No FB & FB & No FB \\
\hline Threshold 50\% & 50.4 & 49.5 & 45.8 & 46.4 & 42.6 & 43.8 \\
\hline $\begin{array}{c}\text { oop one-half } \\
16-84 \% \\
\text { interval }\end{array}$ & 3.03 & 3.13 & 2.85 & 3.03 & 3.68 & 3.28 \\
\hline
\end{tabular}

data again indicate that $S / N$ is the main determinant of the threshold for this task and that these derived thresholds are relatively independent of the feedback state.

The ROC curves used above did not account for operator noise. A new set of ROC curves was generated which accounted for operator noise by increasing the standard deviation of the noise and signal-plus-noise distribution by 3.15. A normal approximation to the discrete distributions was used to generate these new ROC curves. When the new curves are entered with the new thresholds for the corresponding input S/Ns, values of detection and false alarm probabilities are found. The predicted and observed probabilities, as a function of $S / N$, are given in Table 6. The predicted probabilities are extremely close to the empirical probabilities. The application of the construct of operator noise to the noiseless decision process is apparently a good model of the operator's detection behavior. Moreover, the use of the techniques developed above permits an easy comparison between optimum and operator behavior.

Table 6

Obtained Performance and Predicted Performance Bused on Psychometric Functions

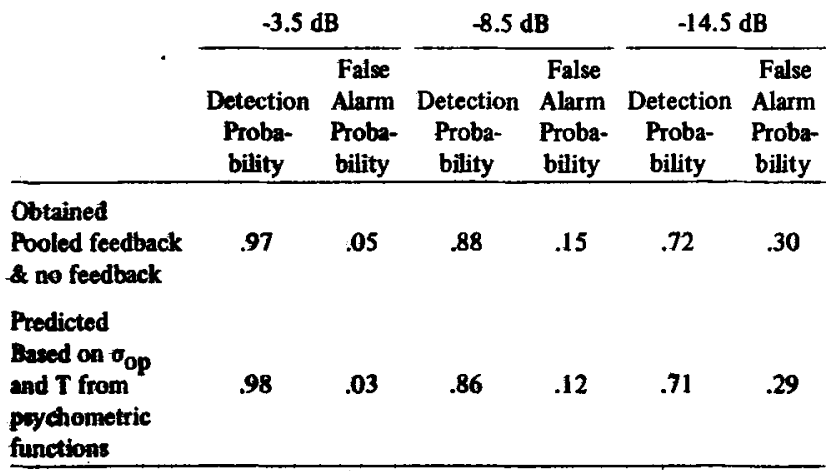

\section{CONCLUSIONS}

The basic purpose of this research was to study operator behavior in a dynamic detection task. In particular, we were concerned with the effects of the parameters of $S / N$ and feedback state. Since the task was such that operator behavior was easily compared to the optimum detector, a model of the operator's behavior was developed with respect to the optimum detector.

The answers to the questions raised about the operator's steady-state responses to a change in stimulus conditions are: (1) The operator behaves as though he has established different thresholds as a function of $\mathbf{S} / \mathrm{N}$. (2) The operator's decision thresholds are relatively independent of the presence or absence of feedback. (3) Given a change from one $S / N$ to another, the operator will change his threshold accordingly.

Although the questions were answered with respect to a "noiseless" decision process, the predictions from the resulting threshold model were systematically biased. The construct of operator noise was introduced to account for the variability added to an equivalent noiseless decision process by the operator. The predicted values of detection and false alarm probabilities from this model were extremely close to the observed values.

\section{REFERENCES}

BOOTH, T. L., GLORIOSO, R. M., LEVY, R. M., WALTER, J. R., \& KAUFMAN, H. M. Displays for studying signal detection and pattern recognition. In Proceedings Spring DECUS Sympostum, New Brunswick, May, 1967.

BRAZEAL, E. H., \& BOOTH, T. L. A high-speed man-computer communication system. In Proccedings Spring DECUS Symposium, Cambridge, May, 1965.

BRAZEAL, E. H., \& BOOTH, T. L. Operator noise in a discrete signal detection task. IEEE Transactions on Human Factors in Electronics, 1966, HFE-7, 164-173.

GREEN, D. M., \& SWETS, J. A. Signal detection theory and psychophysics, New York: John Wiley, 1966.

SELIN, I. Detection theory, Princeton: Princeton University Press, 1965.

NOTES

1. Acknowledgement is due the Office of Naval Research which supported this research through a prime contract (NOnr 2512(00)) with General Dynamics/Electric Boat as a part of the SUBIC (Submarine Integrated Control) program. Portions of this material were presented at Eastern Psychological Association meeting in April 1967 in Boston.

2,3. This research was conducted while the authors were at the Departments of Electrical Engineering and Psychology, respectively, at the Univeraity of Connecticut. The comments of T. L. Booth and H. M. Kaufman are greatly appreciated.

(Accepted for publication Februany 15, 1968.) 